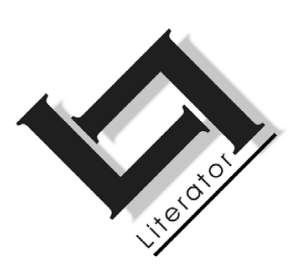

\title{
Trauma in Der Nazi \& der Friseur von Edgar Hilsenrath
}

\author{
J.P.C. van den Berg1 \\ Departement Afrikaans \& Nederlands, Duits \& Frans \\ Fakulteit Geesteswetenskappe \\ Universiteit van die Vrystaat \\ BLOEMFONTEIN \\ E-mail: vdbergjp@ufs.ac.za
}

\section{Abstract \\ Trauma in Der Nazi \& der Friseur by Edgar Hilsenrath}

Theory about trauma and trauma representation can be very useful when interpreting literary texts. The notion of trauma is a complex one and can in general be associated with specific historical experiences, or used as an ontological category to describe fundamental aspects of human existence and identity. From a psycho-analytical perspective the latter refers to a lack within the symbolic order to assimilate and interpret experience in a meaningful way, which consequently leads to a confrontation with the alienating Žižekian real. "Der Nazi \& der Friseur" ("The Nazi \& the barber") by Edgar Hilsenrath deals, among other issues, with the Holocaust experiences of the main character. The aim of this article is to identify possible signs of trauma, both at the level of narrated events and the narration itself. Besides these signs of historical trauma, it will be demonstrated that the play with identity in the novel can be read against the background of trauma used as ontological category, grounded in the "other", as something existing beyond the boundaries of the symbolic order.

1 This material is based upon work supported financially by the National Research Foundation. Any opinion, findings and conclusions or recommendations expressed in this material are those of the author and therefore the NRF does not accept any liability in regard thereto. 


\section{Opsomming}

\section{Trauma in Der Nazi \& der Friseur deur Edgar Hilsenrath}

Die teorie rondom trauma en traumarepresentasie kan met vrug gebruik word by die interpretasie van literêre tekste. Trauma is 'n komplekse begrip wat breedweg geassossieer kan word met 'n bepaalde historiese ervaring, óf gebruik kan word as 'n ontologiese kategorie om fundamentele aspekte van die menslike bestaan en identiteit te beskryf. Vanuit 'n psigo-analitiese perspektief dui laasgenoemde op 'n leemte binne die simboliese orde om ervaring sinvol te assimileer en te interpreteer en dit lei tot 'n konfrontasie met die bevreemdende Žižekiaanse "real". "Der Nazi \& der Friseur" ("Die Nazi \& die haarkapper") deur Edgar Hilsenrath tematiseer onder meer die Holocaustervaring van die hooffiguur. In hierdie artikel word gepoog om moontlike tekens van trauma te identifiseer, sowel op die vlak van die handeling, as op die vlak van die vertelling. Buiten tekens van historiese trauma, sal aangedui word dat die spel met identiteit in die roman gelees kan word teen die agtergrond van trauma as ontologiese kategorie, gegrond in die "ander", as geleë buite die grense van die simboliese orde.

\section{Einleitung}

Die Frage nach der literarischen Darstellbarkeit von Trauma gilt schon seit längerer Zeit als wissenschaftlich produktive Fragestellung, weil dabei auf eine grosse Vielfalt von Disziplinen und Problemfeldern Bezug genommen werden muss. Trauma treibt unter anderem die semiotischen Möglichkeiten der sprachlichen Darstellbarkeit auf die Spitze, und ist deswegen nicht als Sonderproblem zu betrachten, das vom philosophischen, sprachlichen, sozialen, psychologischen und literaturwissenschaftlichen Kontext getrennt untersucht werden könnte. Im Roman Der Nazi \& der Friseur von Edgar Hilsenrath, ist die Darstellung des Verhältnisses von Trauma und Identität des traumatisierten Subjekts thematisch wichtig. Dabei scheint der Begriff der Fremde (Fremdartigkeit, Verfremdung, Befremdung) in diesem Verhältnis eine wichtige Brückenfunktion zu erfüllen. Im folgenden soll versucht werden, Hilsenraths Roman unter dem Aspekt dieses Verhältnisses (der Dialektik von Trauma und Identität) zu deuten, und zu zeigen, inwiefern der Begriff der Fremde für die Darstellung von Trauma und Identität in diesem Roman grundlegend ist. 


\section{Trauma}

Wie der literarische Begriff Tragödie ist auch Trauma in der allgemeinen Umgangssprache ein mehr oder weniger diffuser Begriff. 2 Im Kontext der vorliegenden Erörterung sind die folgenden theoretischen Überlegungen wichtig. Trauma ist nicht in einem einzelnen Ereignis lokalisierbar, sondern manifestiert sich dann, wenn das Erlebte die Darstellungsmöglichkeiten einer Person oder Gruppe insgesamt übersteigt und dieses Erlebte deshalb in seiner vollen Bedeutung nicht mehr zu registrieren, darzustellen und an Dritte zu vermitteln ist. Das will nicht heißen, dass alles, was schwer beschreibbar ist, oder gar (so wie die "Fremde") die Darstellungsmöglichkeiten übersteigt, unbedingt auch als traumatisch aufzufassen ist. Jedes Individuum hat laut Freud eine gewisse "Angstbereitschaft" zur Verfügung, die es inm ermöglicht, sich mit einem schreckhaft-fremdartigen Erlebnis auseinanderzusetzen. Wenn allerdings sogar diese endemische "Vorbereitung", die für den produktiven Umgang mit der Wirklichkeit notwendig ist, fehlt, dann ist das solchergestalt Erlebte als Trauma aufzufassen (Freud, 1921:29).

Wichtig ist also nicht, was erfahren wird, sondern die Struktur solcher Erfahrung: die Art und Weise, wie ein Ereignis vom Traumatisierten erfahren wird (Caruth, 1995:4-5). Demzufolge ist die Traumatisierung eines Individuums als ein Bruch in der "normalen" Assimilierung von solchen Erfahrungen aufzufassen, die normalerweise in einem irgendwie noch rationalisierbaren (sprachlichen, sozio-politischen, ideologischen) Deutungskontext stehen. Denn der Deutungskontext ermöglicht schon von Anfang an persönliche und kollektive Erfahrung. Weil die Möglichkeit zu sinnvollem Registrieren, zu sinnvoller Darstellung und Vermittlung nicht mehr vorhanden ist, wird das "traumatische" Ereignis jetzt anderweitig im Bewusstsein abgelagert: das Ereignis bleibt sozusagen unassimiliert. 3 Symptomatische Manifestation dieses Vorgangs ist die sogenannte Post Traumatic Stress Disorder (PTSD), wobei für das "Ich" die Gefahr entsteht, dass es scheinbar nicht mehr in der Lage ist, als Ordnungsprinzip zu funktionieren und "Sinn" zu schaffen.

Die Bedeutungsgeschichte des Begriffs ist interessant. Ursprünglich zur Bezeichnung einer physischen Verwundung gebraucht, wurde der Begriff in den Arbeiten von etwa J.M. Charcot, Pierre Janet und Sigmund Freud zur psychologischen Traumatheorie erweitert. Dies wird dann in der späteren Psychoanalyse und im (Post-) Strukturalismus weitergeführt. Siehe Leys (2000).

3 Siehe auch Janets Unterscheidung zwischen narrativer und traumatischer Erinnerung (Leys, 2000:229, 233-234, 237-239, 247-254). 
Trauma bedeutet also das Unvermögen, die Wirklichkeit sinnvoll zu assimilieren - mit nicht nur erheblichen Konsequenzen für das Innenleben des Individuums, sondern auch mit der Folge, dass die nach außen gerichtete Vermittlung dieser Erfahrung insgesamt unmöglich wird.

\subsection{Trauma als ontologische Kategorie}

Obwohl die oben erwähnte Definition von Trauma auf die Struktur der persönlichen Erfahrung eines historisch festlegbaren Ereignisses hinweist, kann der Traumabegriff auch erweitert werden, um ihm eine ontologische Bedeutung zu geben. Ontologisch heißt hier, dass dem Begriff Trauma eine transzendentale Bedeutung beigemessen wird, um über die spezifisch historische Erfahrung hinaus auch die allgemeine Auseinandersetzung des Individuums mit der Wirklichkeit zu beschreiben und anzudeuten. Es handelt sich dann nicht mehr nur um ein historisches Ereignis wie etwa den Holocaust (historisches Trauma), sondern mehr noch um eine Grunderfahrung menschlicher Existenz. ${ }^{4}$ Aus der Perspektive der Psychoanalyse gilt die menschliche Geburt zum Beispiel als existenzielles Urtrauma, das aus dem Bewusstsein ins Unbewusste verdrängt wird - wie später das, was nicht in das Muster des Deutungskontexts hineinpasst, ebenso als "traumatisch" ausgeschaltet wird.

Insbesondere Žižeks Rezeption der Lacanschen Psychoanalyse ist im Kontext der ontologischen Auffassung von Trauma aufschlussreich. Žižek übernimmt von Lacan die Zergliederung der Psyche in drei Strukturen: das Reale, das Symbolische und das Imaginäre (Žižek, 1993:178). Für die Traumaproblematik hält er vor allem das Reale und das Symbolische für relevant: mit dem Symbolischen wird der (sprachlich artikulierbare) Deutungskontext, der die Wirklichkeit sinnvoll strukturiert, gemeint - es ist das Universum des Wortes, jenes logos, das es ermöglicht, Existenz als sinnvoll zu erfahren, zu begreifen und zu artikulieren (Žižek, 1999b:253). Demgegenüber gibt es aber im Traumaerlebnis auch das Reale, das

4 LaCapra unterscheidet diese zwei Traumaformen als historisches und strukturelles Trauma. $\mathrm{Er}$ ist vehement in seiner Kritik gegen diejenige Theoretiker, die die beiden gleichsetzen, denn seiner Meinung nach ist die Folge, dass mit solcher Einebnung sogar ein historisches Trauma wie der Holocaust an Einzigartigkeit verliert. Er weist auch auf den Unterschied zwischen absence und loss hin, um den Kontrast deutlich zu machen (LaCapra, 1999:712). 
Nichtzudeutende, das Unbeschreibbare, angesichts dessen die Sinngebungen des Symbolischen versagen müssen. $\mathbf{5}$ Das Reale ist in Žižeks Deutung somit "Das Ding", jenes Etwas, das außerhalb des Symbolischen angesiedelt ist und somit die Begrenzungen des Symbolischen aufzeigt:

The Real is thus the disavowed $X$ on account of which our vision of reality is anamorphically distorted; it is simultaneously the Thing to which direct access is not possible and the obstacle which prevents this direct access, the Thing which eludes our grasp and the distorting screen which makes us miss the Thing. (Žižek, 2006:26.) ${ }^{6}$

Das Reale ist somit das Fremde, das Befremdende, oder, im Žižekschen Sinne, auch das Traumatische oder Traumatisierende, also jenes Ertwas, das die herkömmlichen, vertrauten und bekannten Deutungsmuster des Individuums überfordert und deswegen "fremd" und befremdend anmutet. ${ }^{7}$ Denn es ist ja gerade die erschreckende Fremdheit der traumatisierenden Erfahrung, die nicht mit Worten zu fassen ist; und der Versuch, es dennoch darzustellen, ist wie die perspektivistische Umkreisung eines nicht endgültig zu beschreibenden Kerns. Hierin liegt denn auch die Gemeinsamkeit von Trauma als historisch bestimmter Erfahrung einerseits und als ontologischer Kategorie andererseits. Gleichwohl ist der Unterschied zwischen beiden dadurch bestimmt, dass Trauma als ontologische Kategorie nicht historisch festlegbar ist.

Die beiden Deutungen von Trauma spielen eine wichtige Rolle in der Analyse von Identität: ebenso wie historische Erfahrungen die Identität eines Individuums prägen können, so kann auch die ontologische Dimension, ausgelöst durch das Erlebnis der Fremde, für das Selbstbewusstsein des Subjekts relevant sein.

5 "[...] our representations are forever branded by our finitude, [...] the world of intuited objects is never given in its totality - it shifts into the index of the deficiency of the very discursive form" (Žižek, 1993:20; siehe auch 1991:13).

6 Žižeks Arbeit wird geprägt vom Verhältnis zwischen dem Symbolischen und dem Realen (Žižek, 1999a:135-137; 1991:17, 29, 36, 39, 137, 143; Mowitt, 2000:285, 287; Ball, 2000:39).

7 Ich benutze "Die Fremde" hier ganz spezifisch mit Hinweis auf Žižeks Theoretisierung des Realen, nicht unbedingt im Kontext der heuristischen Tradition von Entfremdung. Deswegen mutet der Gebrauch möglicherweise diffus an, wie das Reale auch beschrieben wird. 


\subsection{Identität im Kontext der Traumadarstellung}

Die Frage ist nun, welche Folgen die Struktur der Traumaerfahrung für die Identität des traumatisierten Subjekts beinhaltet. Wenn die eigene, in einer historischen Erfahrung verwurzelte Identität nicht mehr aus dem Symbolischen heraus sinnvoll strukturiert werden kann, fehlen orientierende Berührungspunkte. Van Alphen (1995: 129) betont den Bruch, der sich zwischen der vom Traumaerlebnis verunsicherten Identität des Subjekts und der Unmöglichkeit eindeutiger Traumadarstellung auftut. Die eigene Identität wird infolge des Traumaerlebnisses verfremdet, wird "fremd", beziehungsweise vom Fremden überlagert und kann folglich nicht mehr eindeutig symbolisch gedeutet und dargestellt werden. Im Opfer eines historischen Traumas zeigen sich dann bestimmte Symptome, die psychologisch als PTSD identifizierbar wären.

Van Alphen erwähnt praktische Beispiele für diesen Bruch: nach inm wird die traumatisierte Identität zum Beispiel gekennzeichnet durch unklare, zweideutige Positionen (man ist weder Subjekt noch Objekt der Ereignisse, oder man ist beides zugleich) und durch eine totale Verneinung der Subjektivität (Van Alphen, 1995:129). Traumageschehen und das Vermögen der subjektiven Traumadarstellung beziehen sich dabei ganz deutlich aufeinander. In literarischen Darstellungen des Holocaust wird zum Beispiel immer wieder versucht, die Opferidentität, manchmal sogar die Täteridentität, als Orientierungspunkt im Symbolischen auszuarbeiten, um eine narrative Darstellung des Traumas überhaupt zu ermöglichen. Identität soll dabei als Stützpfeiler im Darstellungsprozess von historischen Traumaerfahrungen funktionieren. Auf diese Weise wird versucht, das Fremde der traumatischen Erfahrung, das heißt, die traumatische Entfremdung der eigenen Identität aus ihrer "symbolischen" Zuordnung, doch wieder symbolisch bedeutungsvoll werden zu lassen.

Demgegenüber kann jedoch auch die ontologische Dimension von Trauma gebraucht werden, um die Struktur der subjektiven Identität zu beschreiben. Žižek interpretiert Identität ätiologisch als im Trauma gründend (Ball, 2000:17). Vorausgesetzt, dass nicht nur die Wirklichkeit, sondern auch die Identität des Subjekts im Symbolischen konstruiert wird, 8 dann bedeutet das Unvermögen des

8 Das passiert durch die Identifikation mit einem Signifikanten oder der Funktion im Symbolischen (Žižek, 1991:163):

That is to say, the withdrawal into self, the cutting-off of the links to the Umwelt, is followed by the construction of a symbolic universe that the subject projects on to reality as a kind of substitute-formation destined 
Symbolischen, die sinngebende Begründung des individuellen Seins darzustellen ebenfalls, dass der latenten Gegenwärtigkeit des traumatischen Realen Tor und Tür geöffnet wird. Denn sofern das Ich der sinngebenden Struktur des Symbolischen beraubt ist, wird es ein Abgrund des Nichts. ${ }^{9}$ In dieser Hinsicht zitiert Žižek öfters eine Aussage Hegels aus der Jenenser Realphilosophie. Laut Hegel (1987:187) ist der Mensch:

[...] diese leere Nacht, dieses leere Nichts, das alles in ihrer Einfachheit enthält, ein Reichtum unendlich vieler Vorstellungen, Bilder, deren keines inm gerade einfällt - oder die nicht[s] als gegenwärtige sind. Dies [ist] die Nacht, das Innere der Natur, das hier existiert - reines Selbst - in phantasmagorischen Vorstellungen ist es ringsum Nacht, hier schießt dann ein blutig Kopf - dort eine andere weiße Gestalt plötzlich hervor, und verschwindet ebenso. Diese Nacht erblickt man, wenn man dem Menschen ins Auge blickt - in eine Nacht hinein, die furchtbar wird - es hängt die Nacht der Welt hier einem entgegen. (Siehe auch Žižek, 1999a:135-137; 1999b: 253-256. $)^{10}$

Die Fremde oder das Befremdende der eigenen Identität liegt in solchem Fall also, wie Trauma, deutlich jenseits der Grenzen der symbolischen Darstellungsmöglichkeiten. Wäre es also möglich, Trauma an sich, ob historisch ob strukturell gedeutet, und Identität, die im Befremdenden des Traumas gründet, darzustellen?

to recompense us for the loss of the immediate, pre-symbolic Real.

(Žižek, 1999b:254.)

9 Das führt zum Begriff der Groteske: Žižek sieht im traumatischen Kern nicht unbedingt etwas konkretes, sonder vielmehr das dialektische Gegenteil, das Fehlen im Symbolischen. Das Symbolische mutet in dem Sinn grotesk an, weil es Identitäten und Identitätstrukturen aufrechthalten, die eigentlich von Bedeutung aus leer sind. Identität wird somit eine groteske leere Maske, die sich in einer grotesken Wirklichkeit befindet.

[S]elf-consciousness has only to become aware of how this Nothingness which appears to a particular Will as an abstract, opposed, external threat coincides with it's own force of negativity; it has to internalise this force of negativity and recognise in it's own essence, the very kernel of its own being. 'Subject' emerges at this very point of utterly meaningless voidance brought about by a negativity which explodes the frame of balanced exchange. (Siehe auch Žižek, 1993:15.) 


\subsection{Die Darstellung von Trauma}

Wenn Trauma, ob als historische Erfahrung ob als ontologische Kategorie, somit als das Unvermögen aufgefasst wird, das traumatische Geschehen in seiner vollen Bedeutung zu erfassen, wird es wichtig, die Möglichkeiten und Grenzen sprachlicher Darstellung von Trauma zu untersuchen. Im allgemeinen sind die literaturkritischen Beurteilungen in diesem Punkt eher negativ, höchstens wird zugestanden, dass es möglich sei, das traumatische Ereignis rückblickend sprachlich anzupassen und zu modellieren, um es im nachhinein wenigstens einigermaßen - aber nicht voll angemessen - deuten zu können. Das Problem der Nichtrepräsentierbarkeit von Trauma kulminiert etwa bei George Steiner (1988) in der Überzeugung, dass Schweigen sowohl im semiotischen wie auch im ethischen Sinn die einzigmögliche Anwort sei. Demgegenüber artikuliert sich eine positive Bewertung des Traumaproblems in dem Vertrauen in die kathartische Wirkung narrativer Darstellung, mittels welcher die Aufarbeitung des Traumas immerhin für möglich gehalten wird. Vielleicht wäre es ergiebiger, diese beiden Auffassungen nicht als Gegensätze zu betrachten, sondern als die extremen Endpunkte eines Kontinuums. Jeder traumabezogene Text (ob literarisch oder historisch) bewegt sich immer auf diesem Kontinuum.

Obwohl nicht ausschließlich aus dieser Perspektive zu lesen, spielt die Auseinandersetzung mit Trauma im Roman Der Nazi \& der Friseur von Edgar Hilsenrath eine bedeutende Rolle. Aspekte der Traumaerfahrung, wie Traumadarstellung, Identität und die Fremde bilden im Roman ein auffallendes Motivgeflecht. Im folgenden soll versucht werden, die oben dargelegten theoretischen Aspekte am Beispiel des Textes weiter zu beleuchten.

\section{Der Nazi \& der Friseur}

Der Roman, Der Nazi \& der Friseur, hat eine interessante Publikationsgeschichte. In den frühen Siebzigerjahren findet das Manuskript von Edgar Hilsenrath, einem Holocaust-Überlebenden des Mogilev-Podolski Ghettos, in Deutschland keinen Verleger, der bereit gewesen wäre, den Roman zu veröffentlichen. In 1971 erschien The Nazi \& the barber in den USA bei dem angesehenen Verleger Doubleday; es folgten Ausgaben in Italien, Frankreich und England. Fast 60 deutsche Verlage lehnten die deutsche Veröffentlichung des Romans ab, bis 1977 endlich der Verleger Helmut Braun, nach der Begegnung mit Hilsenrath auf der Frankfurter Buchmesse im Jahr zuvor, die deutsche Publikation übernahm. 
Der Grund, warum Hilsenraths Text für deutsche Leser fast verloren gegangen wäre, hängt weniger mit der Frage nach der literarischen Qualität zusammen als mit der Art und Weise, wie im Roman der jüdische Mensch und der Holocaust thematisch dargestellt werden. Denn Hilsenraths Roman durchbricht "die Tabuisierung oder Ikonisierung, mit der das Bild der Juden nach 1945 belegt war" (Braun, 2006:175).

\subsection{Handlung}

Der Nazi \& der Friseur ist ein (meistenteils) in der ersten Person erzählter Lebenslauf der Hauptfigur Max Schulz. Die vorgetragenen Bekenntnisse und zuweilen Verschweigungen, Wiederholungen und Widersprechungen, zusammen mit einem immerwährenden selbstreflexiven Kommentar auf die eigene Erzählung, verleihen dem Ganzen die Allüre eines Zeugnisses. Die Erzählperspektive als Filter steht stärker im Vordergrund als das Erzählgeschehene selbst also die Art, wie sein Leben, wie wichtige historische Ereignisse, wie seine eigene Identität beschrieben und gedeutet werden.

Max Schulz wird 1907 in Wieshalle als Sohn eines Dienstmädchens geboren. Zur Zeit seiner Geburt kommt auch Itzig Finkelstein, Sohn eines jüdischen Ehepaars und später Maxens geschätzter Freund, zur Welt. Als seine Mutter wegen ihrer Ausschweifungen die Wohnung ihres Arbeitgebers verlassen muss, ziehen die beiden in den Friseurladen des Anton Slavitzki um, eines Kinderschänders, der Max schon in der ersten Nacht mit Wissen seiner Mutter vergewaltigt und inn die Jahre danach immer wieder verprügelt. Max schließt eine Freundschaft mit Itzig Finkelstein, besucht öfters dessen Haus und geht später zusammen mit inm bei Itzigs Vater in die Lehre, der ebenfalls wie Slavitzki Friseur ist.

Im Zweiten Weltkrieg findet er sich als SS-Soldat im Konzentrationslager Laubwalde relativ unproblematisch mit der Ermordung der Juden ab. Bereits vor der Ankunft in Laubwalde ist er an einer Massenerschießung auf einem Friedhof beteiligt. Hier beginnen bei inm allerdings doch die ersten Schuldgefühle, denn Max ermordet nicht nur Abertausende von Juden, sondern anscheinend auch die ganze Familie Finkelstein, seinen Freund Itzig eingeschlossen.

Bei der Auflösung des Konzentrationslagers gegen Ende des Krieges entflieht Max mit einem Sack (jüdischer) Goldzähne. Im polnischen Wald trifft er (fast wie im Märchen) auf das Haus eines Hutzelweibes, das inm für den Rest des Winters Unterkunft bietet. Zurück in Berlin trifft er als ehemaliger SS-Soldat die wichtige 
Entscheidung, eine andere Identität anzunehmen, und zwar diejenige seines ehemaligen Freundes Itzig Finkelstein. Er lässt sich eine KZ-Nummer tätowieren und auch beschneiden - seine umfassende Kenntnis der Familiengeschichte der Finkelsteins und der jüdischen Sitten aus seiner Kindheit kommt inm dabei gut zustatten. Maxens Karriere auf dem Schwarzmarkt ermöglicht inm die Beziehung zu einer deutschen Gräfin, die mit ihrem leicht verdeckten Antisemitismus in Max scheinbar zionistische Gefühle erweckt: er wandert später illegal nach Palästina aus.

In Palästina setzt Max seine Karriere als Friseur fort. Maxens zionistische Neigungen entwickeln sich zusehends, und er wird Mitglied einer Terrorgruppe, die versucht, mit Terrorangriffen den eigenen Staat zu erkämpfen. Er nimmt unmittelbar nach der Staatsgründung als israelischer Soldat an dem Krieg gegen die Araber teil, wobei er sich eines Tages auf der Fahrt mit einem Jeep verirrt und bis an den Suezkanal vorstösst. Politisch ist das zwar ein Fehltritt, aber militärisch wird Max als jüdischer Held gefeiert.

Es folgt eine gesellschaftlich erfolgreiche Zeit, aber dann erfolgt eine Wiederbegegnung mit dem Amtsgerichtsrat Wolfgang Richter, einem ehemaligen Bekannten von Max. Richter hat damals mit Max gewettet, dass er den Fall des SS-Schergen Max Schulz aufklären wird und behauptet, dass Max Schulz doch damals von den Partisanen getötet worden sei. Max verneint aber diese Version der Geschichte und gesteht sogar seine echte Identität als Max Schulz, indem er die wahre Version erzählt. Ihm wird zwar nicht geglaubt, aber Max veranlasst Richter doch zu einem Scheinprozess, in dem er, als Max, der Angeklagte ist. Er wird von Richter freigesprochen, weil Gerechtigkeit und Abbüßung für so viele Tote nicht möglich sei.

Am Ende des Romans erlebt Max anlässlich eines (vermeintlichen?) Herzinfarkts in einer Vision den eigenen Tod: eine Herzverpflanzung scheint notwendig zu sein, aber auch die kann ihm das Leben nicht retten. Kurz vor dem Ende hört er die Stimme von Wolfgang Richter, der Maxens Erfahrung von Angst beschreibt:

'Ja. Das ist so. Zuallererst, da stirbt ein Kerl wie du ... mit "ihrer" Angst.'

'Wessen Angst?'

'Mit der Angst deiner Opfer, bevor sie starben.'

'Soll das die gerechte Strafe sein?'

'Nein.' (Hilsenrath, 2004:465.)

Max kann plötzlich wieder sehen und noch im Sterben spürt er, wie der Wind aus dem Wald der sechs Millionen ihn wegführt: "Und die 
Flügel packen mich, krallen sich fest an meinen ausgestreckten Armen. Und der Wind erhebt sich, trägt meine Flügel, und auch mich. Irgendwohin. Dorthin!"

\subsection{Trauma in Der Nazi \& der Friseur}

$\mathrm{Zu}$ untersuchen ist im folgenden, inwiefern die eingangs dargelegte Unterscheidung von Trauma einerseits auf Handlungsebene (fabula) wie andrerseits auf Erzählebene (sujet) für die Interpretation von Hilsenraths Roman produktiv sein kann. Wie früher erwähnt, ist der Roman nicht ausschließlich aus dieser Perpektive zu lesen. Weil aber der (erlebte) Holocaust ein zentrales Element der Handlung ist, ist es doch auffallend, in welchem Maße die bisherige Rezeption die im Roman zumindest implizit angelegte Traumaproblematik zu unrecht ignoriert hat.

Der Unterschied zwischen fabula und sujet ist insofern wichtig, weil die Handlung des Romans von einem unzuverlässigen Erzähler dargestellt wird. Deswegen ist die erzählte Geschichte eine Äußerung, die mehr enthält als bloß eine subjektiv gefärbte Erzählperspektive: vom Erzähler offensichtlich bewusst praktizierte Verzerrungen der Geschichte können vom Interpreten nicht außer Acht gelassen werden. Wenn also Aspekte auf der Erzählebene auf Trauma deuten, wäre abschließend zu fragen, ob diese nicht genau mit Absicht vom Erzähler als bloße Topoi in der Erzählung verwendet werden. Die Frage ist, welche Folgerungen dann zu machen sind: wenn Trauma so nebenbei nur als Topos verwendet wird, bedeutet das dann auch, dass, kein Trauma stattgefunden hat?

Wenn aber doch von genuinem Trauma auszugehen ist, wäre es wichtig zu beachten, welche Art Trauma gemeint ist. Das Trauma des Holocaust als historisch festlegbarem Ereignis, oder Trauma bloß als ontologische Kategorie, um das Trauma der menschlichen Existenz zu demonstrieren? Sollte letzteres der Fall sein, könnte argumentiert werden, dass in der Romanhandlung der Bezug zum historischen Holocaust (möglicherweise) als bloßer Topos verwendet wird. Auch wenn die Traumaproblematik in Der Nazi \& der Friseur eine Vermischung beider Definitionen nahezulegen scheint, so bleibt das sich daraus ergebende Spiel mit der Identitätsfrage für die Interpretation relevant.

Wenn man also als Leser die erzählte Welt von Max Schulz betritt, den Regeln des Erzählers folgt und den Erzähler Max beim Wort 
fasst, wäre der nächste Schritt, auf der Handlungsebene Beweise eines historisch erlebten Traumas aufzusuchen.

\subsubsection{Historisches Trauma}

Es gibt verschiedene Figuren im Roman, die in Maxens subjektiver Erzählung als Opfer des Holocaust ganz deutlich Traumasymptome aufweisen. So etwa hört Max zum Beispiel auf dem Schiff bei der Ausreise nach Palästina noch im nachhinein die Traumaschreie seiner Opfer:

Ich schlafe nicht viel. Wache oft auf ... werde aufgeweckt von gellenden Schreien. Was sagst du, Itzig? Was fragst du? Warum manche Leute schreien ... in der Nacht ... warum sie schreien? - Das sind die Überlebenden, lieber Itzig, die aus unseren Konzentrationslagern. Ich weiß nicht, warum sie noch schreien, obwohl doch längst alles vorbei ist. Vielleicht bloß Angstträume? Ich weiß es wirklich nicht [...] (Hilsenrath, 2004:259).

Wenn Traumatisierung andrerseits als die Unfähigkeit gedeutet wird, Erfahrungen zu artikulieren, weil die sprachlichen Möglichkeiten dazu zu sehr begrenzt sind oder überhaupt ganz fehlen, ist Mina, die Frau von Max, ein geeignetes Beispiel. Als Überlebende einer Massenerschießung 1941 und später als KZ-Häftling, redet sie seit ihren Kriegserfahrungen kein Wort mehr. Als Ersatz für diese kommunikative Hemmung verwandelt sie sich in eine Fressmaschine. Der Beginn von Minas Aufarbeitungsprozess erfolgt dann später bei der offiziellen Proklamation des israelischen Staates, so als ob alle Hemmungen plötzlich aufgehoben würden und sie sich nun auch sprachlich mit ihrer traumatischen Vergangenheit auseinandersetzen könnte. 11

Diese zwei Beispiele folgen der Struktur von der Erfahrung eines historischen Traumas: eine unbeschreibbare Erfahrung, die sich eindeutig auf ein historisches Ereignis bezieht, den Holocaust, die zur gewissen psychologischen Symptomatik führt. Als Nebenfiguren in der narrativen Konstruktion des Erzählers spielen sie aber keine wichtige Rolle.

11 Wenn der Text gelesen wird gegen die Absicht des Erzählers, könnten auch diese Beispiele bloss als "ritualisierte" Strategien des Erzählens aufgefasst werden. Mehr darüber später. 


\subsubsection{Der traumatisierte Max ...?}

Wie aber steht es um die erzählte Figur des Max Schulz (auf der Handlungsebene, fabula), aus der Perspektive des erzählenden Max Schulz? Tätig als SS-Scherge und "mitbeteiligt" an der Ermordung Abertausender von Juden, werden bei Max als erzählter Figur schon zur Zeit seines ersten Einsatzes Symptome deutlich, die auf eine traumatische(?) Reaktion auf das Geschehen schließen lassen. Er meint einen Herzinfarkt zu erleiden, muss dann aber später hören, dass es gar keiner war:

Ich erzählte Veronja während der Liebespausen von meinem Herzinfarkt ... in Russland ... erzählte ihr von den Massenerschießungen ... und das war vor Laubwalde ... das war in Russland ... in Südrussland ... Einsatzgruppe $D$ im südrussischen Abschnitt ... sagte zu Veronja: 'Ich bekam einen Herzinfarkt. Ich war das nicht gewohnt ... damals noch nicht ... so viele auf einmal zu erschießen ... und Frauen und Kinder ... und die Augen ... die Augen, Veronja ... das war zuviel ... und ich dachte, es wäre nur vom Rauchen und Saufen ... aber es waren die Augen. Und ich hatte einen Herzinfarkt, Veronja [...]' (Hilsenrath, 2004:157-158).12

Und genau aus dem späteren ärztlichen Befund, der Infarkt sei eingebildet gewesen, wäre die einzige Folgerung, dass Max als Mörder, als Täter von seinen Erfahrungen im Krieg traumatisiert war. Max scheint nämlich von den Symptomen her von Schuld- und Angstgefühlen heimgesucht. Diese werden im Romantext durchgehend mit zwei Motiven assoziiert: dem Augenmotiv und dem Motiv der sechs Millionen. Neben der wiederholten Erwähnung der Froschaugen als Maxens äußerlichem Identitätsmerkmal wird inm auch die Erinnerung an die Augen der Opfer ständig zur psychologischen Qual. Aber der Kern und Mittelpunkt seiner Schuld und Angst findet sich in Maxens Mitwissen um die Ermordung der sechs Millionen, die für inn durch die von den Juden in Palästina gepflanzten Bäume personifiziert werden: "Ich sage: 'Ja. Die jungen Bäume. Jeder junge Baum ist die Seele eines Toten"' (Hilsenrath, 2004:323). Insbesondere im Wald der sechs Millionen sieht Max sich mit der verdrängten Vergangenheit konfrontiert, die inm als Mitbeteiligten bekannt und doch im gewissen Sinn auch unbekannt 
ist. Der Wald zwingt inn zu Selbstgesprächen, insbesondere in Bezug auf die eigene Schuld. Dabei wird die falsche jüdische Identität, die Max sich angeeignet hat, entlarvt, und Max wird als der "Letzte unter den Letzten" beschrieben: "Weil du dich nicht bekennst! Weil du verleugnest! Und dich versteckst! Und noch dazu: hinter den Opfern ... den toten und denen, die überlebt haben!' (Hilsenrath, 2004:433). Und als Max schließlich in der Todesvision (als "Gerechtigkeitsstrafe") die Ängste seiner Opfer noch im nachhinein miterlebt und nachempfindet, wird er von dem Wind aus dem Wald fortgetragen, "Dorthin!".

Und es kommt mir vor, als käme der Wind aus dem Wald der 6 Millionen. Der Wind! Und der Wind packt die weißen Gardinen vor meinem Fenster. Und schüttelt sie. Und allmählich werden sie dunkler. Die Gardinen am Fenster. Werden dunkler und dunkler, haken sich los, werden zu Flügeln, schwarzen Flügeln, fangen zu flattern an, lassen sich tragen vom Wind, vom Wind, der aus dem Walde kam, dem Wald der 6 Millionen. Und die Flügel packen mich, krallen sich fest an meinen ausgestreckten Armen. Und der Wind erhebt sich, trägt meine Flügel, und auch mich. Irgendwohin. Dorthin! (Hilsenrath, 2004:465.)

An sich bewirken die Stimmen aus dem Wald der sechs Millionen die Entlarvung der von Max erzählerisch konstruierten Scheinidentität als Jude und überlebendes KZ-Opfer. Die Stimmen deuten auf die verdrängten Gefühle, die sich schon früher in den Symptomen des vermeintlichen Herzinfarkts manifestiert haben.

\subsection{3 ... oder nicht?}

Aber der Roman ist schließlich seine Erzählung, sein Zeugnis: und da der Text meistens in der ersten Person erzählt wird, gibt es außer dem Max Schulz/ltzig Finkelstein als erzählte Figur auch den Max Schulz als Erzähler (auf der Ebene des sujets). Die demonstrative Verleugnung der Holocaustwirklichkeit durch nachträglich konstruierte Geschichten und (Schein)-Identitäten - wie auch die im Walde der sechs Millionen ausgelösten schweren Schuldund Angstgefühle - dies alles ist offensichtlich als erlebtes Trauma zu interpretieren. Hingegen, ob Max als Romanfigur tatsächlich selbst traumatisiert ist oder ob das erzählte Trauma nicht vielmehr nur im nachhinein als Erzählung konstruiert wird, ist eine ganz andere Frage. Aus dem Umstand, dass der Erzähler Max zum Beispiel in der Lage ist, sich selbst zu artikulieren, zudem sogar dem Itzig Stimme und Identität zu rauben vermag, meint Gilman (1986/ 1987:145; 1996:123) folgern zu können, dass Max, als erzählendes 
Subjekt, mitnichten traumatisiert sei. Solch eindeutige Interpretation verkennt jedoch die Komplexität der in Hilsenraths Roman enthaltenen Fragestellung. Diese ist im folgenden näher zu untersuchen, denn die Antwort auf die Frage nach der tatsächlichen Traumatisiertheit Maxens gründet sich auf die Frage, in welchem Maß die Erzählung als manipuliertes "Zeugnis" eines unzuverlässigen Erzählers gelesen, oder in welchem Maß sie als authentisches Zeugnis gedeutet wird. Wenn sie eine manipulierte Erzählung, im Gegensatz zu einem authentischen Zeugnis ist, bedeutet das, dass der Erzähler sich gewisser narrativer Strategien bedient, um eine Illusion von Trauma darzustellen. Warum? Um sich als Opfer darzustellen und sich damit von der eigenen Täterschaft loszusprechen? Aber ist dann nicht zu vermuten, dass er dennoch (als Erzähler) heimgesucht wird von der Vergangenheit? Und gibt es dann nicht letztendlich doch noch eine Spur von Trauma als différence?

Es ist so, dass der Erzähler Max sich mit seinem Zeugnis oder seiner Erzählung viel Unrechtmäßiges aneignet: zum Beispiel die Stimme, Geschichte und Identität von Itzig Finkelstein, den er auch getötet hat. Oder um es generisch zu betrachten: als Täter eignet er sich die Opferidentität des Itzig an und als Deutscher die jüdische Identität. Er tut das aber als Erzähler und vermittelt dem Leser somit seine Interpretation der Opfer- und der jüdischen Identität. Die "neue" Identität, die Max sich als Erzähler aneignet, ist nach wie vor nichts anderes als die auf Vorurteile reduzierte, vollständige Stereotypisierung des Juden. Denn Max übernimmt im Grunde nur Äußerlichkeiten, zunächst die tätowierte KZ-Nummer und die Beschneidung (mit seinen Froschaugen meint er ohnehin bereits dem stereotypisierten Klischee zu entsprechen). Danach erlernt er jüdische Gebräuche, die jüdische Geschichte und sogar die Sprache, dies alles aber nur zum äußeren Schein, ohne dadurch auch im Wesen jüdisch sein zu können. Der Roman enthält somit den Versuch des Erzählers Max, mittels narrativer Darstellung die Vergangenheit aus seiner Erzählperspektive (mit allen erzählerischen Unzuverlässigkeiten) darzustellen. Er bestimmt die Form der Repräsentation und die Darbietung wie auch die Festlegung von Handlung und Figuren.

Die literarische Form, die zur Darstellung der Geschichte (sujet) verwendet wird, ist das Zeugnis. An sich ist das Zeugnis als literarische Textsorte, im Vergleich zu anspruchsvoll durchkomponierten Texten, formal und inhaltlich relativ unbefangen. Die für selbstdarstellende Zeugnistexte typischen Aspekte wie Wiederholungen und 
Lücken, die eine gewisse Brüchigkeit zur Folge haben, sind Merkmale, die auch in Der Nazi \& der Friseur anwesend sind. Wegen der innen innewohnenden Brüchigkeit eignen sich Zeugnistexte insbesondere für die Darstellung von Trauma, eine Darstellungsform, die denn auch in Maxens Erzählung genau so instrumentalisiert wird. Solche "Instrumentalisierung" wird vom Autor mit Absicht gebraucht, denn er gebraucht andererseits auch wieder gewisse narrative Strategien, die genau als Gegenteil der unbefangenen Aussage funktionieren und vielmehr auf zielbewusste literarische Formgebung hinweisen. Folglich entsteht eine Spannung zwischen (scheinbar unbefangenem) Zeugnis und (absichtsvoll literarischer) Manipulation, und dadurch wird das Ganze in der Schwebe gehalten. Ohne hier näher auf Einzelheiten einzugehen, kann gesagt werden, dass der Romantext deutlich kalkulierten literarischen Eingriffen unterliegt. Der Erzähler Max gebraucht zum Beispiel Motive aus den Märchen, um seine Geschichte zu gestalten und um Figuren und Handlungen in solchem Raster festzulegen (Taylor, 1995:440-441; Arnds, 2002:432-433). Es gibt ein Spiel mit Gattungen und Gattungsmerkmalen (z.B. dem Bildungsroman und AntiBildungsroman); auch führt das Spiel mit Stereotypen, Übertreibungen und Simplifizierungen, das letztendlich ein hinterfragendes Spiel mit dem Wissen um das Symbolische ist, deutlich in die Welt der Groteske. Dies alles deutet unbedingt auf eine gezielte Manipulation der Geschichte, wodurch die Frage in Bezug auf die Authentizität der Traumatisierung des Erzählers offen bleibt. Die usurpierte Identität von Max muss auch in diesem Kontext gesehen werden. Die Frage ist, ob hier die Darstellung eines konkreten historischen Traumas obwaltet, oder ob solchergestalt manipulierte Traumadarstellung nicht eher als heuristisches Mittel zur Beschreibung der ontologischen Dimension verstanden werden muss - wenn von Trauma überhaupt noch die Rede sein kann.

Allerdings wird des Erzählers narrativ so eindeutig konstruierte Geschichte und (Schein)-Identität im Wald der sechs Millionen Bäume schließlich doch als wesenlose Fassade entlarvt. Es scheint somit Max unmöglich zu sein, sich den eigenen Schuldgefühlen zu entziehen: in der Erwähnung des Baummotivs - und vollends am Ende des Romans mit dem Verschwinden ins unbekannte "Dorthin" - werden Risse in der scheinbaren Eindeutigkeit der Darstellung sichtbar. Es gelingt dem Erzähler Max nicht, das Erzählte in einen konsistenten Zusammenhang zu bekommen, und in den Momenten, wo das Raster seiner Repräsentation sich als unzulänglich erweist, sickern die Gegenstimmen aus dem Wald der sechs Millionen durch. 
Ob nun die Figur des Max traumatisiert ist oder nicht, das vom Erzähler Max abgelegte Zeugnis als solches ist in seiner Unzulänglichkeit von Verschweigungen, Wiederholungen und Widersprechungen selbst eine eindrucksvolle Artikulation des angedeuteten Holocaust-Traumas - das in diesem Fall als Täter-Trauma dem OpferTrauma zur Seite gestellt wird. Bestätigt wäre somit die eingangs erwähnte Definition des Traumas als unrepräsentierbar, als nicht lokalisierbar in den gängigen narrativen Konventionen mit deren eindeutigen Identitätsdifferenzierungen von Opfer und Täter, Deutscher und Jude, Herrenmensch und Untermensch und so weiter. Indem die Erzählung jede eindeutige Erklärung oder Sinngebung vermeidet, entspricht sie genau der eingangs erwähnten Definition des Wesens von Trauma.

Hinzu kommt, dass [Hilsenrath] in seinem Roman Erklärungen für das Geschehene weitestgehend verweigert und sich auch nicht auf eine Sinnsuche begibt, die verzweifelt versucht, das Sinnlose sinnhaltig $\mathrm{zu}$ machen. Dies ist der entscheidende Unterschied zu den Werken anderer Shoaautoren [...] (Braun, 2006:197).

Es sei denn, dass die ganze Erzählung als die raffinierte Illusion eines literarisch strukturierten Zeugnisses aufgefasst wird.

\subsubsection{Trauma als ontologische Kategorie: Identität und Trauma}

Für die Erzählerfigur Max ist die Aneignung einer falschen und konstruierten Identität, der Identitätswechsel vom antisemitischen Spießer zum vorbildlichen Bürger des Staates Israel, freilich eine unbedingt notwendige Überlebensstrategie. Die ostentative Betonung seiner arischen Herkunft und Identität am Anfang des Romans entspringt einerseits den Minderwertigkeitsgefühlen der Familie Finkelstein gegenüber, aber dann auch den Misshandlungen durch den Stiefvater, inklusive der als Baby erlittenen Vergewaltigungen. Die Frage ist nun, wie der spätere Identitätswechsel beziehungweise Identitätsraub sich vollzieht? Die einfachste Antwort findet sich in dem Rat, den die adlige Kriemhild Gräfin von Hohenhausen Max gibt: "Was du ererbt von deinen Vätern hast, erwirb es, um es zu besitzen.' Ein Goethezitat. Merken Sie sich das!" Und Max merkt es sich, obwohl er die konstruierte jüdische Identität gerade 
nicht von seinen Vätern ererbt hat, und vielmehr in diesem Punkt alles erwerben muss. ${ }^{13}$

Im Verwandlungsprozess integriert Max zwar die stereotypisierten äußerlichen Merkmale jüdischer Identität (das Symbolische), keineswegs aber den eigentlichen, im Grunde auch gar nicht bestimmbaren Kern (das Reale). Als ob Identität reduzierbar wäre auf rein äußerlich-kognitive Kenntnisse von Riten, Sitten, Gebräuchen und auf soziopolitische und gesellschaftliche Funktionen und Konventionen. In Bezug auf Maxens Versuch, sich Itzig Finkelsteins Identität als Jude und Opfer fälschlich anzueignen, fehlen ihm zunächst zwei wichtige äußere Zeichen, die aus seiner Sicht eindeutig auf diese Identität hinweisen: die Beschneidung als Zeichen des Juden und die KZ-Tätowierung als Zeichen des Opfers. Es handelt sich dabei aber lediglich um die fassadenhafte Aneignung von Riten und Zeichen, deren wirkliche existenzielle Konsequenz Max am eigenen Leibe nicht erlebt hat und deren wahre Bedeutung er folglich weder begreifen noch würdigen kann (siehe Gerstenberger \& Pohland, 1992:82). ${ }^{14}$

Insofern macht der Roman deutlich, dass Identität nicht auf solch äußere Art und Weise entstehen kann. Schon die in der Kindheit auffallende (äußerliche) Verwechselbarkeit von Max und Itzig betont zwei Grundeinsichten: erstens die Einmaligkeit subjektiv-echter Identität (die Tatsache, dass echte Identität immer stereotypisierte Grenzen übersteigt und somit unvermeidlich auch "fremd" ist) und zweitens die Austauschbarkeit der Klischees und Stereotypisierungen von gesellschaftlich bestimmten, rein vordergründig-äußerlichen Identitätsmerkmalen. Die Austauschbarkeit von stereotipisierten Identitäten und Begriffen ist denn auch ein Grundmotiv des Romans: Opfer und Täter, Deutscher und Jude, Herrenmensch und Untermensch, Gewinner und Verlierer, sexuelle Identität und sprachliche Identität (siehe Gilman, 1996:122),15 Philosemitismus

13 Der Wechsel ist also nicht wie die Verwandlung eines Zauberkünstlers, sondern tatsächlich die Aneignung einer konstruierten Identität und Geschichte.

14 Stereotypisierungen von gängigen Identitätsmerkmalen, z.B. von dem Juden, dem Opfer, dem Täter, dem Untermenschen, dem Herrenmenschen unsoweiter, entstehen in verschiedenen Kontexten (siehe Hilsenrath, 2004:9, 30-31, 44-45, 55-56, 69; Gilman, 1996:122, 123).

15 Maxens zionistische Redeführungen im Friseursalon in Beth David sind fast verwechselbar mit der früher beschriebenen Redeführung von Hitler auf dem Ölberg (Hilsenrath, 2004:365, 368-369). 
und Antisemitismus, Schuld und Unschuld, unsoweiter. Die Austauschbarkeit dieser Stereotypen betont inre relative Zufälligkeit, und gerade darin liegt eine wesentliche Aussage der narrativen Darstellung. Das hat zur Folge, dass auch die narrativ vorgetäuschte Eindeutigkeit der Traumarepräsentation in diesem Roman weitgehend in Unsicherheit gerät. Darüberhinaus gibt die Austauschbarkeit oder Umkehrung der Rollen dem Text, im Kontext des historischen Holocausttraumas, eine ausgesprochen groteske Note, die von der gängigen Rezeption als ethische Zumutung gewertet wird. Das Holocausttrauma als humoristische Groteske!

Max versteht zwar die fremde Identität, die er sich aneignen muss, aber es ist inm nicht möglich, mit dem, was er zu verstehen meint, identisch zu werden. Er beobachtet sie von außen und versucht, sie mit vorgeprägten Auffassungen zu deuten, aber sie entschlüpft inm wie ein Geruch, der nicht festzuhalten ist. Sogar die "eigene" Identität ist inm schwer beschreibbar, wenn er im Spiegelbild eine Unmenge an Möglichkeiten sieht, sodass im Spiegel selbst das eigene Ich fremd anmutet. Wie ist diese "Fremde" in der eigenen Identität, und darüber hinaus in der Identität des Anderen zu deuten? Hier muss wieder verwiesen werden auf den Gegensatz zwischen der im Symbolischen begründeten Identität und der Dominanz jenes Realen, das sich jeder symbolischen Deutung entzieht - im gewissen Sinn die hegelsche Nacht der Welt. Folglich kann in diesem Kontext von einem nichtrepräsentierbaren "traumatischen" (Identitäts-) Kern geredet werden, wenn Trauma als ontologische Kategorie gedeutet wird. Natürlich führt diese ätiologische Auffassung von der Strukturierung der Identität bei weitem nicht zu einer psychologischen Symptomatik (PTSD), wie das bei der traumatischen Erfahrung eines historisch festlegbaren Ereignisses der Fall ist. Aber das Befremdende des Nichtrepräsentierbaren spielt hier eine ebenso wichtige Rolle.

Und wenn der Erzähler dann im Prozess gegen Ende des Romans mit dem Geständnis "Herr Amtsgerichtsrat. Ich bin Max Schulz!" gegen seine Symbolisch angeeignete, falsche Identität aufbegehrt, ist folglich die Reaktion des Richters gegenüber seiner Frau, "Ihr Mann ist verrückt geworden!" (Hilsenrath, 2004:451), nichts anderes als konsequent. Identität ist auch in diesem Urteil wieder Symbolisch gesellschaftlich bestimmt, und der Versuch, das Gegenteil zu behaupten, gilt als Wahnsinn. Für die "Fremde", die jenseits der herkömmlich festgestellten Identitätsstrukturen des Ichs und des Anderen wahre Identität konstituiert, ist kein Platz, es sei denn als Wahnsinn. 
Maxens Zeugnis wird so zum Zeugnis des Unvermögens, sich im Kontext festgelegter Symbolischer Grenzen, wie sie im sozio-politischen Raster vorgegeben sind, die Fremde einer anderen Identität aneignen zu wollen.

Schreiben über den Holocaust findet sich in einem besonderen Dilemma wieder: einerseits gilt es, die Wiederherstellung zerstörter Identität leisten zu müssen, andererseits aber gilt es gleichermaßen zu vermeiden, durch Anknüpfung an vergangene Identitäten auf Modelle zurückzugreifen, die ihre Tragfähigkeit im Zuge komplexer historischer Veränderungen eingebüßt haben. (Gerstenberger \& Pohland, 1992:91.)

\section{Fazit}

Als Ergebnis der Untersuchung ist somit festzuhalten, dass der Roman als so interpretiertes Zeugnis eine den herkömmlichen Leser fremd anmutende Unassimilierbarkeit des historischen HolocaustTraumas zum Ausdruck bringt. Dem Ich-Erzähler Max ist es nämlich nicht möglich, auf der Erzählebene eine eindeutige narrative Repräsentation zu vermitteln, die die traumatische Erfahrung des tatsächlichen (historischen) Holocaust nachvollziehbar artikulieren könnte. Die Stimmen aus dem Wald der sechs Millionen wirken zwar als Korrektur von Maxens vexativ konstruierter Version, sind aber in ihrer Traumaerfahrung von Maxens teils bewusstem teils unbewusstem Rückbezug auf diese abgekoppelt. Auch die schematische Festlegung von klar abgegrenzten Identitäten in Gegensatzpaaren ist nicht möglich, da diese fortwährend verwechselt und relativiert werden.

Als manipuliertes Zeugnis, als literarischer Text, der sich verschiedener narrativer Strategien bedient, scheint die Erzählung einerseits bewusst eine Traumatisiertheit bei Max (dem Erzähler) zu suggerieren, wohingegen die literarische Manipulation andererseits eigentlich jedwelches Trauma bei Max (der erzählten Figur auf der Handlungsebene) leugnet. Es sei denn, man wäre bereit, auch das manipulierte Zeugnis als verzögerte authentische Traumadarstellung (différence) aufzufassen.

Ob man die Darstellung des historischen Holocausttraumas nun für die Romanaussage für relevant hält oder nicht - die ontologishe Bedeutung der Traumadarstellung, die in der Fremdartigkeit jenseits des Symbolischen gründet, ist in Maxens zeugnishaftem Lebensbericht sehr deutlich präsent. Folglich wirkt die Fremde, indem es das Symbolische jeglicher Sinngebung beraubt, als gemeinsamer 
Nenner zwischen den beiden Möglichkeiten der Auseinandersetzung mit der Traumaproblematik.

Denk mal nach, Itzig. Schon aus diesem Grund hätt' ich euch hassen müssen. Um zu verleugnen, was ich gar nicht bin ... bloss, weil ich Angst hatte, ich könnte es sein. Oder: weil sie glaubten, dass ich es bin, obwohl ich wusste, dass ich es nicht bin. Kapierst du das? (Hilsenrath, 2004:244.)

Die Traumaproblematik, historisch oder ontologisch gedeutet, ist in Der Nazi \& der Friseur ein Schlüsselthema. Ob im Kontext historisch festlegbarer Erfahrungen gebraucht, oder als ontologische Beschreibung des menschlichen Seins jenseits dessen symbolischer Darstellung - die Auseinandersetzung mit der gesamten Traumaproblematik könnte als heuristisch ergiebiege Interpretationsstrategie interessante Perspektiven auf die hermeneutische Erschließung literarischer Texte eröffnen.

\section{Literaturverzeichnis}

ARNDS, P. 2002. On the awful German fairy tale: breaking taboos in representations of Nazi euthanasia and the Holocaust in Gunter Grass's Die Blechtrommel, Edgar Hilsenrath's Der Nazi und der Friseur, and Anselm Kiefer's visual art. The German quarterly, 75(4):422-439.

BALL, K. 2000. Trauma and its institutional destinies. Cultural critique, 46:1-44.

BRAUN, H. 2006. Ich bin nicht Ranek: Annäherung an Edgar Hilsenrath. Berlin: Dittrich Verlag.

CARUTH, C. 1995. Introduction. (In Caruth, C., ed. Trauma: explorations in memory. Baltimore: Johns Hopkins University Press. p. 3-12.)

FREUD, S. 1921. Jenseits des Lustprinzips. Leipzig: Internationaler Psychoanalytischer Verlag.

GERSTENBERGER, K. \& POHLAND, V. 1992. Der Wichser: Edgar Hilsenrath - Schreiben über den Holocaust, Identität und Sexualität. Der Deutschunterricht, 44:74-91.

GILMAN, S.L. 1986/1987. Jewish writers and German letters: anti-semitism and the hidden language of the Jews. The Jewish quarterly review, New Series, 77(2 \& 3): 119-148.

GILMAN, S.L. 1996. Hilsenrath und Grass Redivivus. (In Kraft, T., Hrsg. Edgar Hilsenrath: das unerzählbare Erzählen. München: Piper. S. 119-127.)

HEGEL, G.W.F. 1987. Jenaer Systementwürfe. TI. 3: Naturphilosophie und Philosophie des Geistes. Hamburg: Meiner Verlag.

HILSENRATH, E. 2004. Der Nazi \& der Friseur. Berlin: Dittrich Verlag.

LACAPRA, D. 1999. Trauma, absence, loss. Critical inquiry, 25(4):696-727.

LEYS, R. 2000. Trauma: a genealogy. Chicago: University of Chicago Press.

MOWITT, J. 2000. Trauma and its cultural aftereffects. Cultural critique, 46:272297. 
STEINER, G. 1988. The long life of metaphor: an approach to the "Shoah". (In Lang, B., ed. Writing and the Holocaust. New York: Holmes \& Meier. p. 154-174.)

TAYLOR, J. 1995. Writing as a revenge: reading Edgar Hilsenrath's Der Nazi und der Friseur as a Shoah survivor's fantasy. History of European ideas, 20(1-3):439-444.

VAN ALPHEN, E. 1995. De holocaust en de grenzen van representatie. (In Bulhof, I. \& Van Riesen, R., reds. Als woorden niets meer zeggen: de crisis rond woord en beeld in de huidige cultuur. Kampen: Kok. p. 123142.)

ŽIŽEK, S. 1991. Looking awry: an introduction to Jacques Lacan through popular culture. Cambridge: Massachusetts Institute of Technology Press.

ŽIŽEK, S. 1993. Tarrying with the negative: Kant, Hegel, and the critique of ideology. Durham: Duke University Press.

ŽIŽEK, S. 1999a. Otto Weininger; or, Woman doesn't exist. (In Wright, E. \& Wright, E., eds. The Žižek reader. Oxford: Blackwell. p. 127-147.)

ŽlŽEK, S. 1999b. Schelling-in-itself: the orgasm of forces. (In Wright, E. \& Wright, E., eds. The Žižek reader. Oxford: Blackwell. p. 251-267.)

ŽIŽEK, S. 2006. The parallax view. Cambridge: Massachusetts Institute of Technology Press.

\section{Kernbegrippe:}

Hilsenrath, Edgar

Holocaust

identiteit

trauma

traumarepresentasie

\section{Key concepts:}

Hilsenrath, Edgar

Holocaust

identity

representation of trauma

trauma 